\title{
A boost for the EW SUSY hunt: monojet-like search for compressed sleptons at LHC14 with $100 \mathrm{fb}^{-1}$
}

\author{
Alan Barr ${ }^{a}$ and James Scoville ${ }^{a, b}$ \\ ${ }^{a}$ Department of Physics, Denys Wilkinson Building, \\ Keble Road, Oxford, OX1 3RH U.K. \\ ${ }^{b}$ United States Air Force Institute of Technology, \\ 2950 Hobson Way, Wright-Patterson Air Force Base, OH, 45433 U.S.A. \\ E-mail: A.Barr1@physics.ox.ac.uk, james.scoville@physics.ox.ac.uk
}

AbSTRACT: Current Large Hadron Collider (LHC) analyses are blind to compressed supersymmetry (SUSY) models with sleptons near the lightest super partner (LSP) in mass: $m_{\tilde{l}}-m_{\tilde{\chi}_{1}^{0}} \equiv \Delta m \lesssim 60 \mathrm{GeV}$. We present a search sensitive to the very compressed range $3 \mathrm{GeV}<\Delta m<24 \mathrm{GeV}$ using the channel $p p \rightarrow \tilde{l}^{+} \tilde{l}^{-}+$jet $\rightarrow l^{+} l^{-} \tilde{\chi}_{1}^{0} \tilde{\chi}_{1}^{0}+$ jet with soft same-flavor leptons and one hard jet from initial state radiation $\left(p_{\mathrm{T}}^{j}>100 \mathrm{GeV}\right)$. The sleptons recoil against the jet boosting them and their decay products, making the leptons detectable and providing substantial missing transverse momentum. We use the kinematic variable $m_{\mathrm{T} 2}$ along with a different-flavor control region to reduce the large standard model backgrounds and control systematic uncertainty. We find the analysis should allow LHC14 with $100 \mathrm{fb}^{-1}$ to search for degenerate left-handed selectrons and smuons in the compressed region up to $m_{\tilde{l}_{L}} \lesssim 150 \mathrm{GeV}$. In addition, it should be sensitive to $m_{\tilde{l}_{L}} \lesssim 110 \mathrm{GeV}$ for the very challenging case of auto-concealed SUSY, in which left-handed sleptons decay to the Kaluza-Klein tower of a modulino LSP which lives in $d=6$ extra dimensions. In both the compressed spectrum and auto-concealed SUSY scenarios this analysis will need more data to improve on LEP2 limits for right-handed sleptons due to their smaller cross sections.

Keywords: Supersymmetry Phenomenology, Hadronic Colliders

ArXiv EPrint: 1501.02511 


\section{Contents}

1 Introduction 1

2 Simulation 2

2.1 Tools 2

2.2 Slepton signal 3

2.3 Backgrounds 4

3 Beating the backgrounds 5

3.1 Key variables: $m_{\tau \tau}^{2}$ and $m_{\mathrm{T} 2}$

3.2 Analysis cuts 6

$\begin{array}{ll}3.3 \text { Controlling background systematics: SF-DF } & 10\end{array}$

4 LHC14 reach with $100 \mathrm{fb}^{-1} \quad 11$

5 Conclusions 13

\section{Introduction}

Discovering supersymmetry (SUSY) at the Large Hadron Collider (LHC) requires distinguishing the signal decay products of SUSY particles (sparticles) from large standard model (SM) backgrounds. In $R$-parity conserving SUSY in which the lightest super partner (LSP) is stable and invisible to the detector, methods commonly used to separate signal from backgrounds take advantage of the signal's large missing transverse momen$\operatorname{tum}\left(E_{\mathrm{T}}^{\mathrm{miss}}\right)$, numerous jets from visible final state radiation, or highly energetic leptons [1]. However, this becomes more challenging in the case of electroweak (EW) SUSY production where cross sections are modest, and is especially difficult for compressed EW SUSY scenarios in which the mass difference between the produced sparticle and the LSP is small. Compressed SUSY scenarios result in less energetic final state radiation and less $E_{\mathrm{T}}^{\mathrm{miss}}$. Not only are such processes harder to pick out of backgrounds during an analysis, but they may not even pass trigger requirements for the LHC detectors. As a result, both CMS and ATLAS remain blind to compressed EW SUSY scenarios involving sleptons with $m_{\tilde{l}}-m_{\tilde{\chi}_{1}^{0}} \equiv \Delta m \lesssim 60 \mathrm{GeV}[2,3]$.

One way to circumvent the challenges of compressed SUSY is to search for events with an energetic jet from initial state radiation (ISR) [4-7]. In such events the sparticles will recoil against the ISR, increasing both the energy of visible decay products and $E_{\mathrm{T}}^{\text {miss }}$. Such a strategy was used, for instance, by both ATLAS and CMS to search for top squarks with masses close to the LSP $[8,9]$. However monojet searches are typically not designed to find EW sparticles and therefore those analyses veto on leptons to suppress unwanted 
backgrounds. Recently, several groups investigated the possibility of using a monojet, large $E_{\mathrm{T}}^{\text {miss }}$, and soft leptons to pick out Higgsinos in a compressed spectrum [10-12]. Motivated by their strategy and the current absence of slepton limits from the LHC for compressed models, we investigate the possibility of performing a similar search to discover sleptons.

We rely on a hight- $p_{\mathrm{T}}$ ISR jet to boost pair produced same flavor opposite sign (SFOS) sleptons, which promptly decay to two neutralino LSPs and a lepton pair $\left(p p \rightarrow \tilde{l}^{+} \tilde{l}^{-} j \rightarrow\right.$ $\left.l^{+} l^{-} \tilde{\chi}_{1}^{0} \tilde{\chi}_{1}^{0} j\right)$. Like refs. $[10,11]$ we search for a hard central jet, large $E_{\mathrm{T}}^{\text {miss }}$, and a soft SFOS lepton pair. However, unlike these studies we find it difficult to pick the signal out of the large backgrounds from leptonically decaying $t \bar{t}$ and $W^{+} W^{-} j$ using a veto on large $m_{l l}$ alone; since signal leptons come from opposite legs of the decay chains their angular separation is not always small. Therefore we use the leptons' 'stransverse' mass $m_{\mathrm{T} 2}$ [1315] to compress signal events into a narrow window and make the signal competitive with backgrounds.

However, even this is insufficient to pick out the signal if there is $\mathcal{O}(20 \%)$ systematic uncertainty in the remaining backgrounds. Thus we use a different flavor lepton pair control region (i.e. $e \mu$ ) to subtract away these backgrounds, similar to the CMS study in ref. [16]. With this we are able to investigate the potential exclusion reach of the LHC at $\sqrt{s}=14 \mathrm{TeV}(\mathrm{LHC} 14)$ with $100 \mathrm{fb}^{-1}$ if no excess in signal events are seen.

In addition to the normal compressed spectrum scenario we also consider an autoconcealed (AC) SUSY scenario [17]. In this extra-dimensional SUSY model the slepton can decay to a nearly continuous Kaluza-Klein (KK) tower of neutral states, with a denser number of states closer to the parent mass $m_{\tilde{l}}$. The effect of the multiple states is phenomenological similar at colliders to a compressed spectrum of near-degenerate particles. The result is that current LHC searches are not sensitive to direct slepton production in auto-concealed scenarios [17]. ${ }^{1}$

\section{Simulation}

\subsection{Tools}

We investigated the prospect of discovering slepton production in near-degenerate scenarios at the LHC using simulations of $p p$ at $\sqrt{s}=14 \mathrm{TeV}$, assuming an integrated luminosity of $100 \mathrm{fb}^{-1}$. To generate signal events and SM backgrounds we used MadGraph5_aMC@NLO at tree level [20] paired with Pythia 6 [21] for showering and hadronization. With the exception of taus, decays into leptons were done at the matrix element level (within Madgraph) thus retaining spin correlations and increasing generator efficiency. Taus were decayed using Tauola [22] within Pythia.

\footnotetext{
${ }^{1}$ During the preparation of this paper, two searches were published which also attacked the compressed spectrum slepton problem. The first to do so [18] used vector boson fusion and missing transverse momentum to reduce standard model backgrounds, but required around $3000 \mathrm{fb}^{-1}$ of data. The second [19] proposed a solution remarkably similar to ours, also using a high- $p_{\mathrm{T}}$ monojet to help generate $E_{\mathrm{T}}^{\mathrm{miss}}$ and employing a moving $m_{\mathrm{T} 2}$ window to distinguish signal from background. However some of the details of signal selection differ and in particular they do not address background systematics as we do in this paper using the different flavor control region.
} 
For both the SUSY signal $\left(p p \rightarrow \tilde{l}^{+} \tilde{l}^{-} j\right)$ and the dominant background $(p p \rightarrow t \bar{t})$ we used MLM matching with up to one additional jet (e.g. $p p \rightarrow \tilde{l}^{+} \tilde{l}^{-} j j$ ). For all backgrounds we used a generator level cut $R_{l l} \equiv \sqrt{\Delta \eta^{2}+\Delta \phi^{2}}>1.0$ between leptons and for all nonMLM matched processes we used a generator level cut on the leading jet $p_{\mathrm{T}}>80 \mathrm{GeV}$ to increase efficiency (both of which were looser than the final analysis level cuts and were checked to not impact the results).

Events were then fed into an analysis built by the authors within the program CheckMATE [23-25], which includes an improved ATLAS detector simulation in Delphes [26]. In our analysis, jets and isolated leptons were assigned as follows:

- Jets were defined using the anti- $k_{\mathrm{T}}$ algorithm $[27,28]$ with a distance parameter 0.4 , $|\eta|<4.5$, and $p_{\mathrm{T}}>20 \mathrm{GeV}$. The assumed $b$-jet tagging efficiency is $80 \%$. Further details on the $b$-tagger can be found in ref. [23].

- Isolated leptons were defined to mimic ref. [29]. Electrons (muons) were required to have $|\eta|<2.47, p_{\mathrm{T}}>7 \mathrm{GeV}$ and to not be within $R<0.4$ of a reconstructed jet or $R<0.1$ of another isolated lepton. The $p_{\mathrm{T}}$ sum of tracks above 0.4 (1) GeV within $R<0.3$ was required to be less than $16(12) \%$ of the lepton $p_{\mathrm{T}}$. Electrons had the additional requirement that the sum of energies within $R<0.3$ was required to be less than $18 \%$ of the electron energy.

\subsection{Slepton signal}

We consider two cases: a classic compressed SUSY scenario and an auto-concealed SUSY scenario, described in further detail below. For the classic scenario we use a simplified model with a bino LSP and degenerate selectrons and smuons, both left or right-handed, with all other sparticles decoupled. The sleptons decay with a $100 \%$ branching ratio to a lepton of the same flavor plus the LSP. In this simplified model at leading order slepton production and decay depends on the physical masses and the gauge couplings but not on soft SUSY parameters. Therefore a spectrum generator was not used and we simply set the physical masses of the sparticles to their desired values in the Madgraph parameter card - the masses of the decoupled sparticles were set to $8 \mathrm{TeV}$.

In the auto-concealed SUSY scenario, sleptons are constrained to live on a brane in a $4+d$ dimensional bulk and can decay promptly to a lepton and a nearly continuous tower of bulk LSP KK-modes. The branching ratio to KK-modes with mass $m$ is given by $[17]^{2}$

$$
\frac{d \Gamma}{\Gamma_{\text {tot }}}=\frac{1}{\Gamma_{\text {tot }}} \frac{\Omega_{d}}{(2 \pi)^{d}} \frac{m_{\tilde{l}}^{d+3}}{8 \pi M_{*}^{d+2}}\left(\frac{m}{m_{\tilde{l}}}\right)^{d+1}\left(1-\frac{m^{2}}{m_{\tilde{l}}^{2}}\right)^{2} \frac{d m}{m_{\tilde{l}}}
$$

where $m_{\tilde{l}}$ is the parent slepton mass, $V$ is the bulk volume, $\Omega_{d}$ is the surface area of a $(d-1)$-sphere, $M_{*}$ is the fundamental gravitational scale, and $\Gamma_{\text {tot }}$ is the slepton total decay width. Assuming the relevant scales are such that the slepton decay is prompt, this leads to a quasi-compressed spectrum as shown in figure 1.

\footnotetext{
${ }^{2}$ For decays to a modulino KK-tower. For other LSP cases see [17].
} 


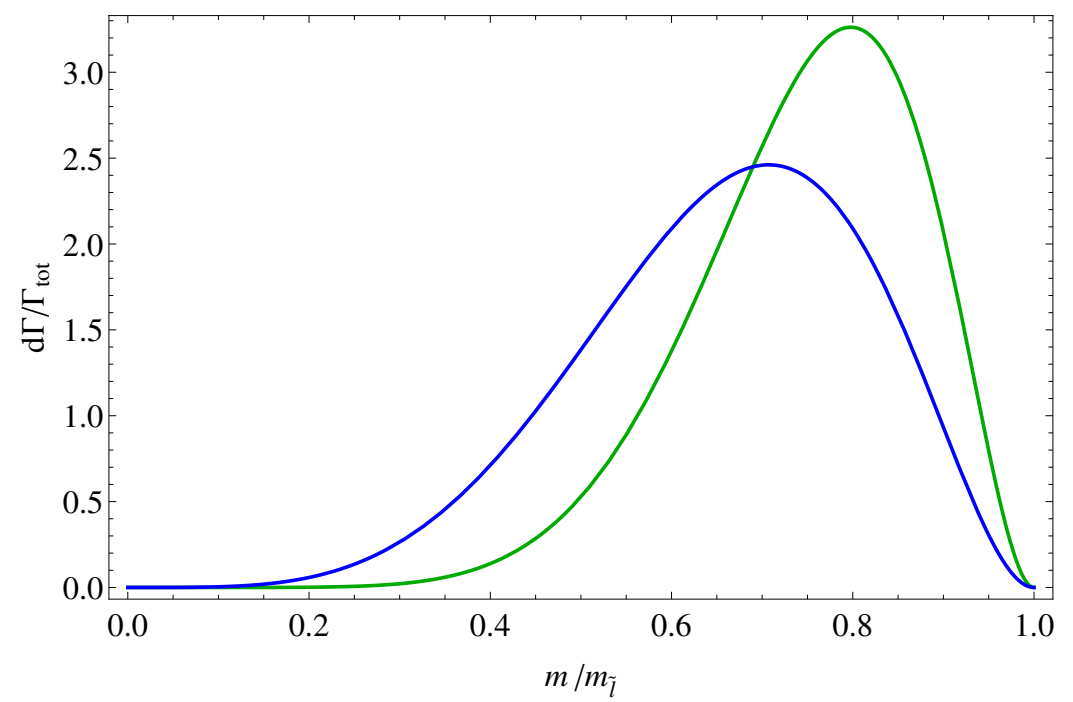

Figure 1. Differential decay rate for sleptons in an auto-concealed SUSY scenario. Here sleptons decay to the KK-tower of a bulk modulino LSP living in $d=6$ (green) or $d=3$ (blue) extra dimensions. The modulino mass is given as a ratio to the slepton mass $m_{\tilde{l}}$ on the horizontal axis. Since the number of KK-modes grows like $\sim m^{d-1}$, the $d=6$ scenario results in a more compressed spectrum.

We approximate the continuous spectrum as in [17] by introducing $N=20$ new gauge neutral spin $1 / 2$ states in Pythia. The masses of these states $m_{j}$ fell into $N$ evenly spaced bins from 0 to the slepton mass $m_{\tilde{l}}$. The mass $m_{j}$ of the $j^{\text {th }}$ state was given by the branching ratio-weighted average of masses in the $j^{\text {th }}$ bin, and the branching fraction to this state was determined by the integrated width over the bin.

\subsection{Backgrounds}

We estimate SM backgrounds in the 2 leptons +1 jet $+E_{\mathrm{T}}^{\text {miss }}$ channel by modelling the processes below. Tops and $W$ 's are decayed leptonically within Madgraph. In all processes except $\left(Z / \gamma^{*}\right) j$, leptonic decays include taus (which are then decayed in Tauola); $\left(Z / \gamma^{*}\right) j$ decaying to taus is included in the $\tau \tau j$ background. The backgrounds were:

1. $\tau^{+} \tau^{-} j$.

2. $t \bar{t}$.

3. $W^{+} W^{-} j$.

4. $l^{+} l^{-}(Z \rightarrow \nu \bar{\nu}) j$ where the leptons are primarily from $Z / \gamma^{*}$. We will simply refer to this process as $Z Z j$.

5. $l^{+} l^{-} W^{ \pm} j$. Despite the fact that this process produces three visible leptons, its relatively large cross section combined with the possibility of losing a lepton makes this background more significant than $Z Z j$. We will refer to this process as $W Z j$. 
6. $t W$ with jets defined to come from light-flavor or $b$-quarks. To increase generation efficiency it is required that the jet $p_{\mathrm{T}}$ be greater than $80 \mathrm{GeV}$.

7. $t q$ with jets defined to come from light-flavor or $b$-quarks and the same jet cut is imposed as in $t W$.

8. $l^{+} l^{-} j$ where the required missing transverse momentum comes primarily from mismeasured jets. We will refer to this process as $Z j$.

We also investigated the rare processes $t \bar{t} W$ and $t \bar{t} Z$ but found contributions from these processes was negligible.

At this point it is worth noting that these backgrounds are similar to what were considered in refs. [10, 11] which looked for Higgsino pair production plus an additional jet, although there are a few differences. First, like ref. [10], we find that backgrounds from single-top and $W Z j$ processes, while sub-dominant, are non-negligible (ref. [11] did not consider these backgrounds). Second, unlike ref. [10], we did not include a $k$-factor enhancement of the $t \bar{t}$ cross section for two reasons (ref. [10] included a NNLO+NNLL enhancement of 1.72). The first is that at least some of this enhancement will come from $t \bar{t}$ events with additional initial state radiation which would then be preferentially rejected by our second-jet veto; thus it is questionable whether one should simply scale up the leading order cross section. The second reason is that we do not include NLO enhancements for any of our backgrounds or our signal, despite the fact that SUSY signal $k$-factors can be substantial. $^{3}$ Clearly, a better option would be to generate both signal and background events at NLO - an option we do not pursue for this first study.

Finally, although neither refs. [10] nor [11] considered the background from $Z j$ plus mismeasured $E_{\mathrm{T}}^{\mathrm{miss}}$ we find that it provides a potentially substantial background, albeit one that is easily dealt with by requiring a large separation $R$ between the leptons and a cut on reconstructed $m_{\tau \tau}^{2}$. Both studies used a $m_{\tau \tau}^{2}$ cut which would have likely dealt with the majority of this background, although their definitions of this reconstructed variable differ from each other as we discuss in section 3 below.

\section{Beating the backgrounds}

\subsection{Key variables: $m_{\tau \tau}^{2}$ and $m_{\mathrm{T} 2}$}

The SM backgrounds discussed in section 2.3 are much greater than the SUSY signal we are seeking. To reduce the BG we use a series of kinematic cuts, including cuts on the reconstructed $m_{\tau \tau}^{2}$ designed to separate the $\tau \tau j \mathrm{BG}$, and on the 'stransverse mass' $m_{\mathrm{T} 2}$, with which we define our final signal windows.

To reconstruct the di-tau invariant mass squared $m_{\tau \tau}^{2}$ we follow ref. [10] and use the fact that taus recoiling against a $100 \mathrm{GeV}$ jet are highly relativistic and their decay products will be nearly parallel. Thus in a fully leptonic di-tau decay we write the sum of the neutrinos' transverse momentums as

$$
\mathbf{p}_{\mathrm{T}}^{\text {miss }}=\xi_{1} \mathbf{p}_{\mathrm{T}}^{l_{1}}+\xi_{2} \mathbf{p}_{\mathrm{T}}^{l_{2}}
$$

\footnotetext{
${ }^{3}$ For instance, ref. [30] found a $k$-factor of 2.3 for neutralino pair + jet production.
} 
where $\mathbf{p}_{\mathrm{T}}^{\text {miss }}$ is the missing transverse momentum vector and $\xi_{n}$ is a scale factor relating the transverse momentums of the $n^{\text {th }}$ tau daughter neutrinos to the transverse momentum of the daughter electron or muon $\mathbf{p}_{\mathrm{T}}^{l_{n}}$. Using this set of two equations we solve for the unknowns $\xi_{1}$ and $\xi_{2}$, with which we find the four-momenta of the taus: $p_{\tau_{n}}=\left(1+\xi_{n}\right) p_{l_{n}}$. Then the di-tau invariant mass squared is

$$
m_{\tau \tau}^{2}=2\left(1+\xi_{1}\right)\left(1+\xi_{2}\right) p_{l_{1}} \cdot p_{l_{2}} .
$$

This definition differs from ref. [11] in that it allows for a negative invariant mass squared if $\xi<-1$ for a single $\xi$. From eq. (3.1) we see this can occur for a missing transverse momentum vector nearly opposite to a lepton's $\mathbf{p}_{\mathrm{T}}$ and $\mathrm{p}_{\mathrm{T}}^{\text {miss }}>\mathrm{p}_{\mathrm{T}}^{l}$. For example, this may happen in $W W j$ when a neutrino and a lepton (possibly coming from different $W$ decays) are nearly back-to-back. Though having a negative invariant mass squared is clearly unphysical-which motivates ref. [11] to scale energy by $\left|\xi_{i}\right|$ rather than $\xi_{i}$-we do not concern ourselves with this fact since the approximation $\mathbf{p}_{\mathrm{T}}^{\nu_{1}} \approx \xi_{1} \mathbf{p}_{\mathrm{T}}^{l_{1}}$ is not always valid and in practice we find this definition slightly more effective at separating signal from background.

To determine the final signal regions we use the kinematic variable $m_{\mathrm{T} 2}$, defined as

$$
m_{\mathrm{T} 2}\left(p_{\mathrm{T}}^{l_{1}}, p_{\mathrm{T}}^{l_{2}} ; m_{\tilde{\chi}_{1}^{0}}\right)=\min _{q_{\mathrm{T}}}\left[\max \left(m_{\mathrm{T}}\left(p_{\mathrm{T}}^{l_{1}}, q_{\mathrm{T}} ; m_{\tilde{\chi}_{1}^{0}}\right), m_{\mathrm{T}}\left(p_{\mathrm{T}}^{l_{2}}, p_{\mathrm{T}}^{\mathrm{miss}}-q_{\mathrm{T}} ; m_{\tilde{\chi}_{1}^{0}}\right)\right)\right]
$$

where $m_{\tilde{\chi}_{1}^{0}}$ is the mass of the neutral particle which produces $E_{\mathrm{T}}^{\text {miss }}$ and

$$
m_{\mathrm{T}}\left(p_{\mathrm{T}}^{l}, q_{\mathrm{T}} ; m_{\tilde{\chi}_{1}^{0}}\right)=\sqrt{m_{l}^{2}+m_{\tilde{\chi}_{1}^{0}}^{2}+2\left(E_{\mathrm{T}}^{l} E_{\mathrm{T}}^{q}-\mathbf{p}_{\mathrm{T}} \cdot \mathbf{q}_{\mathrm{T}}\right)} .
$$

In a two-body semi-invisible decay, the stransverse mass variable $m_{\mathrm{T} 2}$ provides an event-by-event bound in the space of masses of the parent and the invisible daughter particle. In practice, it is usually employed as a function which takes as an input the invisible particle's proposed mass, and returns the maximal lower bound on the mass of the parent particle. For example, in a pair-decay of sleptons of mass $m_{\tilde{l}}$ to leptons and LSPs of mass $m_{\tilde{\chi}_{1}^{0}}$ the function $m_{\mathrm{T} 2}\left(m_{\tilde{\chi}_{1}^{0}}\right)$ is always smaller than $m_{\tilde{l}}$ when the correct LSP mass is input. Since the same is not necessarily true for background processes, we can preferentially select the signal by imposing a requirement that $m_{\mathrm{T} 2}\left(m_{\tilde{\chi}_{1}^{0}}\right)<m_{\tilde{l}}$ for our trial LSP and slepton masses. In this way, we can scan the $m_{\tilde{l}}-m_{\tilde{\chi}_{1}^{0}}$ plane.

\subsection{Analysis cuts}

Using the variables $m_{\tau \tau}^{2}$ and $m_{\mathrm{T} 2}$ as defined above we use the following analysis cuts to pull the slepton signal out of the much larger SM backgrounds which also produce 2 leptons + 1 jet $+E_{\mathrm{T}}^{\mathrm{miss}}$ :

1. Veto events with a tagged $b$-jet to reduce backgrounds involving tops.

2. Require one hard central jet with $|\eta|<2.5$ and $p_{\mathrm{T}}>100 \mathrm{GeV}$. Veto events with a second jet with $|\eta|<4.5$ and $p_{\mathrm{T}}>40 \mathrm{GeV}$. 


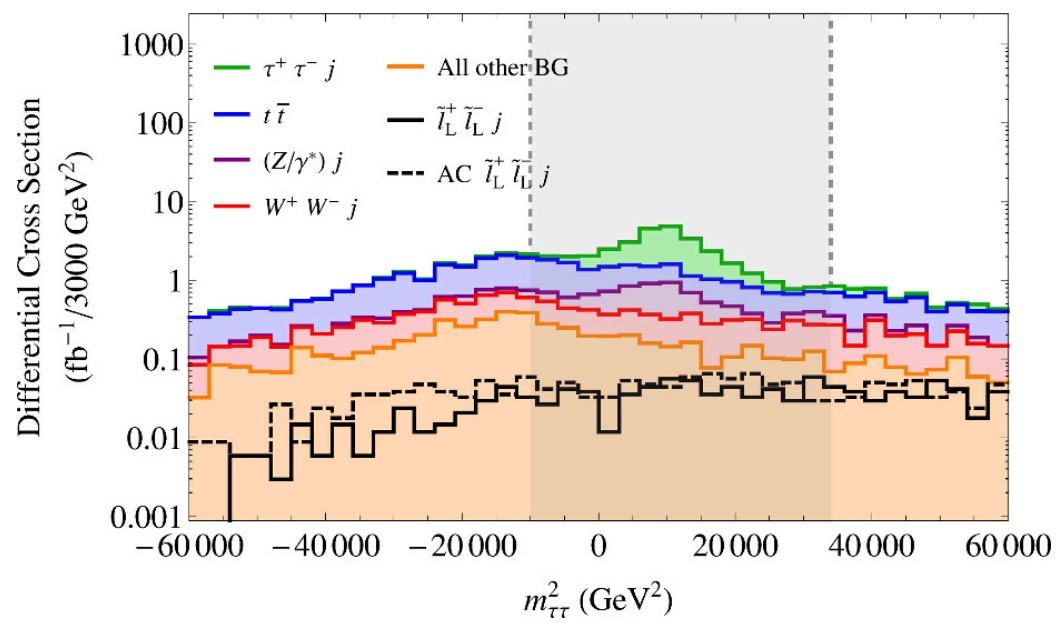

Figure 2. Reconstructed $m_{\tau \tau}^{2}$ distributions after cuts 1-4 for backgrounds (histograms stacked) and two different signal scenarios: degenerate left-handed selectrons and smuons with mass $m_{\tilde{l}_{L}}=$ $104 \mathrm{GeV}$ each decaying to a lepton plus either a $96 \mathrm{GeV}$ neutralino LSP (solid black) or an autoconcealed modulino KK-tower (dashed black). The gray area indicates the region removed in step 5 of the analysis. This cut significantly reduces the $\tau \tau j$ and $Z j$ backgrounds.

3. Require $E_{\mathrm{T}}^{\mathrm{miss}}$ of at least $100 \mathrm{GeV}$. Also require $|\Delta \phi|>1.5$ between the hard jet and $\mathbf{p}_{\mathrm{T}}^{\text {miss }}$. This helps to reduce backgrounds from $Z j$ with $E_{\mathrm{T}}^{\text {miss }}$ coming from mismeasured jet energy.

4. Require two SFOS isolated leptons with $R>1.3$ between them. The large $R$ requirement significantly reduces backgrounds involving $Z / \gamma^{*}$ (in particular the otherwise large $W Z j$ background in which one lepton escapes detection) and is more effective than a soft invariant mass veto since this would also veto signal leptons which tend to be soft.

5. Veto events with a reconstructed $m_{\tau \tau}^{2}$ between $-10,000 \mathrm{GeV}^{2}<m_{\tau \tau}^{2}<34,000 \mathrm{GeV}^{2}$. This is very effective at reducing the $\tau \tau j$ background and also helps pare down $Z j$. The $m_{\tau \tau}^{2}$ distributions of backgrounds and representative signal models are shown in figure 2 .

6. Require $\min \left(R_{j l}\right) / E_{l}>0.02$ where $\min \left(R_{j l}\right)$ gives the minimum distance $R$ between the hard jet and the leptons and $E_{l}$ is the energy of the lepton closest to the jet. Cutting small $R_{j l}$ helps to isolate tops since their $b$-jets are closer to their leptons than the decay products from signal sleptons, which recoil against the hard ISR jet. This is more effective than an invariant mass cut on $\min \left(m_{j l}\right)$ since leptons from compressed spectra decays are softer than leptons from top/ $W$ decays and this partially compensates for the closer distance between the jet and lepton. We take advantage of this fact by dividing $R_{j l}$ by the lepton energy, further differentiating tops from signal while also cutting into the $W W j$ background. The $\min \left(R_{j l}\right) / E_{l}$ distributions of backgrounds and representative signal models are shown in figure 3 . 


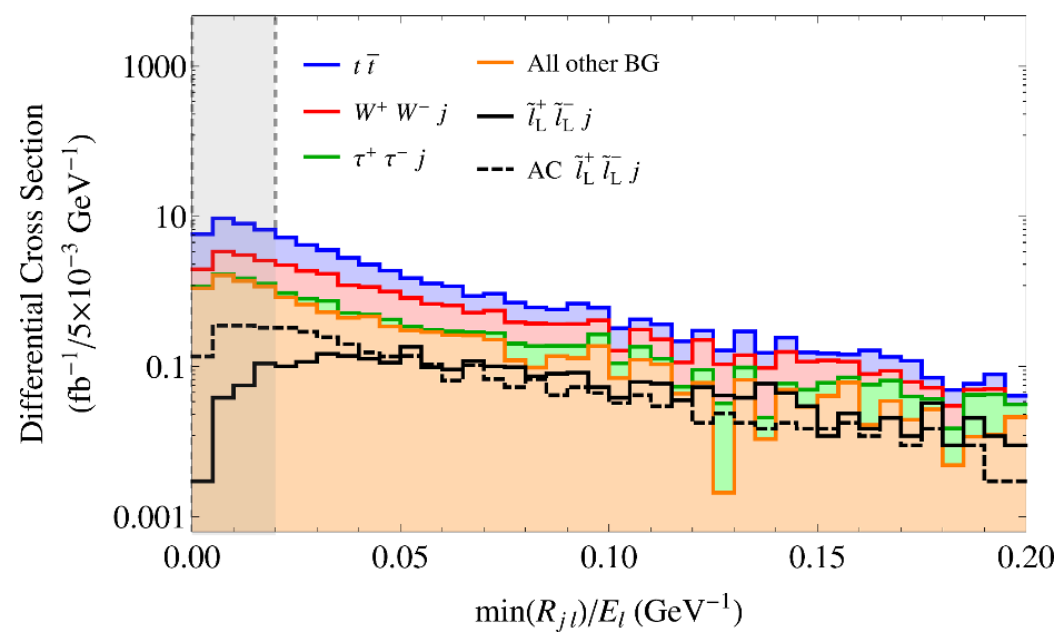

Figure 3. $\min \left(R_{j l}\right) / E_{l}$ distributions after cuts $1-5$ for backgrounds (histograms stacked) and two different signal scenarios: degenerate left-handed selectrons and smuons with mass $m_{\tilde{l}_{L}}=104 \mathrm{GeV}$ each decaying to a lepton plus either a $96 \mathrm{GeV}$ neutralino LSP (solid black) or an auto-concealed modulino KK-tower (dashed black). The gray area indicates the region removed in step 6 of the analysis.

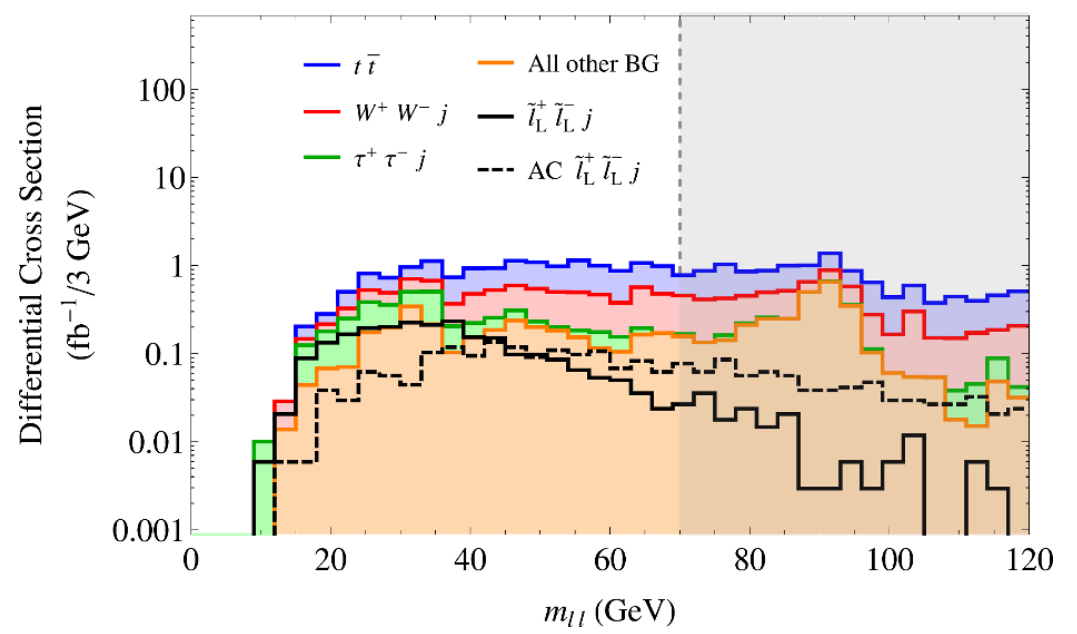

Figure 4. $m_{l l}$ distributions after cuts 1-6 for backgrounds (histograms stacked) and two different signal scenarios: degenerate left-handed selectrons and smuons with mass $m_{\tilde{l}_{L}}=104 \mathrm{GeV}$ each decaying to a lepton plus either a $96 \mathrm{GeV}$ neutralino LSP (solid black) or an auto-concealed modulino KK-tower (dashed black). The gray area indicates the region removed in step 7 of the analysis.

7. Require the two leptons' invariant mass $m_{l l}<70 \mathrm{GeV}$. This cuts away hard leptons from top $/ W$ decays. While cutting even lower on $m_{l l}$ can slightly extend the mass reach of the search, this comes at the expense of weakening the reach for larger $\Delta m$, since sleptons further separated from the LSP will have harder leptons. The $m_{l l}$ distributions of backgrounds and representative signal models are shown in figure 4 .

8. The final signal regions are defined using the stransverse mass as a function of the trial LSP mass $m_{\mathrm{T} 2}\left(\tilde{\chi}_{1}^{0}\right)$ as discussed in section 3.1 , requiring $m_{\mathrm{T} 2}\left(\tilde{\chi}_{1}^{0}\right)$ is less than 


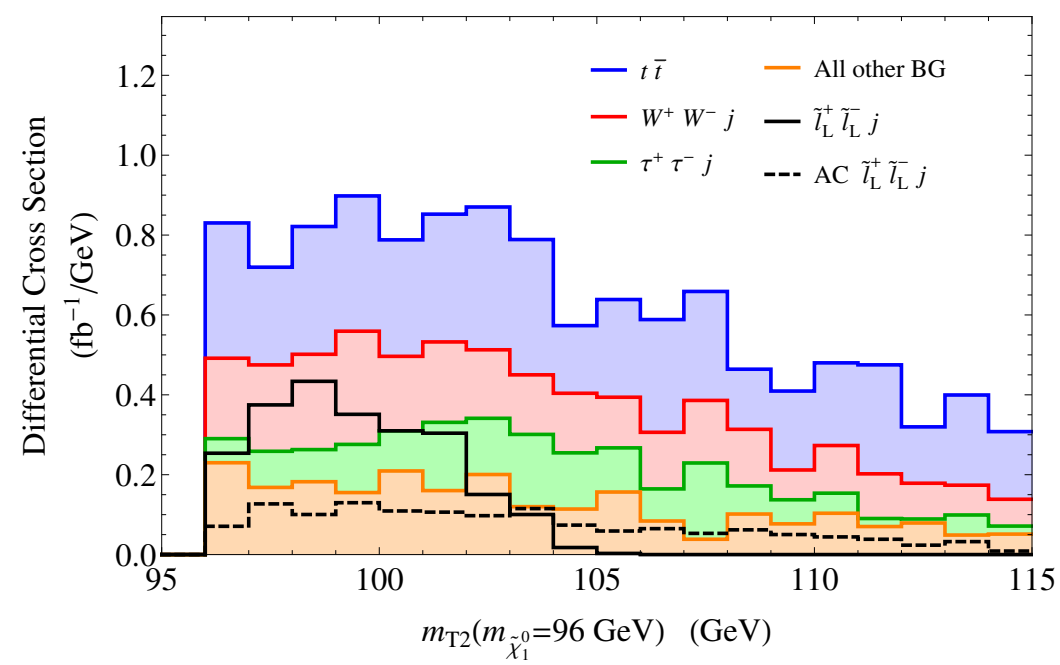

Figure 5. $m_{\mathrm{T} 2}\left(m_{\tilde{\chi}_{1}^{0}}\right)$ distribution after cuts 1-7 for backgrounds (histograms stacked) and signal using $m_{\tilde{\chi}_{1}^{0}}=96 \mathrm{GeV}$ for both. Note that the vertical axis is not log-scaled. Two different signal scenarios are shown: degenerate left-handed selectrons and smuons with mass $m_{\tilde{l}_{L}}=104 \mathrm{GeV}$ each decaying to a lepton plus either a $96 \mathrm{GeV}$ neutralino LSP (solid black) or an auto-concealed modulino KK-tower (dashed black). When scanning the $\left(m_{\tilde{l}}, m_{\tilde{\chi}_{1}^{0}}\right)$ plane the expected signal and backgrounds at $\left(m_{\tilde{l}}, m_{\tilde{\chi}_{1}^{0}}\right)=(104,96) \mathrm{GeV}$ are evaluated in step 8 using $m_{\mathrm{T} 2}(96 \mathrm{GeV})<104 \mathrm{GeV}$. For the auto-concealed model, where multiple states play the role of LSP, we use three bins to evaluate the signal $-m_{\mathrm{T} 2}(80 \mathrm{GeV}), m_{\mathrm{T} 2}(88 \mathrm{GeV}), m_{\mathrm{T} 2}(96 \mathrm{GeV})<104 \mathrm{GeV}-$ and choose the one with the largest signal significance.

the trial $m_{\tilde{l}}$. In this way, we scan the $m_{\tilde{l}}-m_{\tilde{\chi}_{1}^{0}}$ plane. To evaluate the auto-concealed model, where multiple states play the role of LSP, we use three bins to evaluate the signal $-m_{\mathrm{T} 2}\left(m_{\tilde{l}}-24 \mathrm{GeV}\right), m_{\mathrm{T} 2}\left(m_{\tilde{l}}-16 \mathrm{GeV}\right), m_{\mathrm{T} 2}\left(m_{\tilde{l}}-8 \mathrm{GeV}\right)<m_{\tilde{l}}-$ and choose the one with the largest signal significance. The $m_{\mathrm{T} 2}$ distribution for $m_{\tilde{\chi}_{1}^{0}}=96 \mathrm{GeV}$ is shown in figure 5 .

Cutflows for the background and representative signal samples are show in table 1. Unfortunately, even after cuts $1-8$ a significant number of background events from $t \bar{t}$, $W W j$, and $\tau \tau j$ remain, as can be seen in figure 5 . Since we will evaluate the signal significance as $s=S / \sigma_{B}$ where $S$ is the expected number of signal events and $\sigma_{B}$ is the total background uncertainty, even a moderate $\sigma_{B}(\mathcal{O}(20 \%))$ will overwhelm the signal. If we use

$$
\sigma_{B}=\sqrt{B+\sigma_{B \text { sys }}^{2}}
$$

where $\sigma_{B \text { sys }}$ is the systematic uncertainty of the background, and $B$ is the expected number of background events, ${ }^{4}$ then it is clearly necessary to have good control over background systematics to obtain a detectable signal significance. We attack this problem in the next section.

\footnotetext{
${ }^{4}$ Here and in what follows we will use the Gaussian limit to approximate Poisson statistical errors as $\sigma_{B \text { stat }}=\sqrt{B}$.
} 


\begin{tabular}{|l|c|c|c|c|c|c|c|c|c|}
\hline & $\tau \tau$ & $t \bar{t}$ & $W W j$ & $Z Z j$ & $W Z j$ & $t W$ & $t q$ & $Z j$ & $l_{L}^{+} l_{L}^{-} j$ \\
\hline MG cuts & 3769013 & 5077488 & 74580 & 3478 & 21820 & 158000 & 2497581 & 4955268 & 10590 \\
\hline 1) $b$-veto & 3167916 & 902842 & 61618 & 2883 & 18187 & 38262 & 795944 & 4240902 & 9302 \\
\hline 2) hard jet & 417284 & 56546 & 19345 & 866 & 4548 & 8296 & 87754 & 1342713 & 1845 \\
\hline 3) $E_{\mathrm{T}}^{\text {miss }}$ & 130135 & 25823 & 9855 & 478 & 1723 & 2784 & 46904 & 46654 & 1477 \\
\hline 4) SFOS $l l$ & 1751 & 4423 & 1726 & 186 & 289 & 553 & 169 & 570 & 327 \\
\hline 5) $m_{\tau \tau}^{2}$ & 230 & 3511 & 1438 & 144 & 229 & 443 & 141 & 209 & 269 \\
\hline 6) $R_{j l_{1}} / E_{l}$ & 196 & 1613 & 905 & 75 & 141 & 171 & 137 & 115 & 248 \\
\hline 7) $m_{l l}$ & 174 & 713 & 397 & 8 & 25 & 85 & 113 & 60 & 229 \\
\hline 8) $m_{\mathrm{T} 2}$ & 94 & 256 & 164 & 3 & 8 & 32 & 67 & 30 & 227 \\
\hline
\end{tabular}

Table 1. Background and signal counts for $p p$ collisions with $\sqrt{s}=14 \mathrm{TeV}$ and an integrated luminosity of $100 \mathrm{fb}^{-1}$. This table shows the number of events which pass the Madgraph (MG) generator level cuts described in section 2.3 and analysis cuts $1-8$. The $m_{\mathrm{T} 2}$ cut in this instance requires that $m_{\mathrm{T} 2}(96 \mathrm{GeV})<104 \mathrm{GeV}$. This corresponds to the signal model $\left(m_{\tilde{l}}, m_{\tilde{\chi}_{1}^{0}}\right)=(104,96) \mathrm{GeV}$. The far right column shows the cutflow for the matching model with degenerate left-handed selectrons and smuons with mass $m_{\tilde{l}_{L}}=104 \mathrm{GeV}$ decaying to a SFOS lepton pair plus two $96 \mathrm{GeV}$ neutralinos.

\subsection{Controlling background systematics: SF-DF}

To reduce background systematics we estimate the size of the expected background using a different flavor (DF) di-lepton control region containing $e+\mu$ events which pass the equivalent of cuts 1-8. Since sleptons are produced in same flavor (SF) opposite sign pairs only, while the dominant backgrounds produce different flavor lepton pairs as often as same flavor pairs, the DF control sample can be used to estimate-and hence subtract-the majority of the remaining backgrounds.

To do so we use a background subtraction scheme similar to that used in a CMS BSM search [16]. Inside each signal region, we multiply the number of events in the DF control region $B_{\mathrm{DF}}$ by a normalization factor $n$ to account for the different efficiencies in detecting muons and electrons. We then subtract the number of normalized control region events from the number of background signal region events $B_{\mathrm{SF}}$ to obtain the final background count: $B=B_{\mathrm{SF}}-n B_{\mathrm{DF}}$. The residual systematic uncertainty in the corresponding number of background events should now come from uncertainty in $n$, which was determined to within $4 \%$ in the CMS study. We therefore take this as an estimate of our systematic uncertainty. This comes at the cost of inflating the statistical uncertainty since the total background uncertainty is now

$$
\begin{aligned}
\sigma_{B} & =\sqrt{\left(\frac{\partial B}{\partial B_{\mathrm{SF}}}\right)^{2} \sigma_{\mathrm{SF} \mathrm{stat}}^{2}+\left(\frac{\partial B}{\partial B_{\mathrm{DF}}}\right)^{2} \sigma_{\mathrm{DF} \mathrm{stat}}^{2}+\left(\frac{\partial B}{\partial n}\right)^{2} \sigma_{n}^{2}} \\
& =\sqrt{B_{\mathrm{SF}}+n^{2} B_{\mathrm{DF}}+B_{\mathrm{DF}}^{2} \sigma_{n}^{2}}
\end{aligned}
$$

where $\sigma_{\mathrm{SF} \text { stat }}$, and $\sigma_{\mathrm{DF} \text { stat }}$ are the statistical uncertainties in $B_{\mathrm{SF}}$ and $B_{\mathrm{DF}}$ respectively and $\sigma_{n}=0.04 n$ is the total uncertainty in $n$ assumed in this study. Despite the increased 


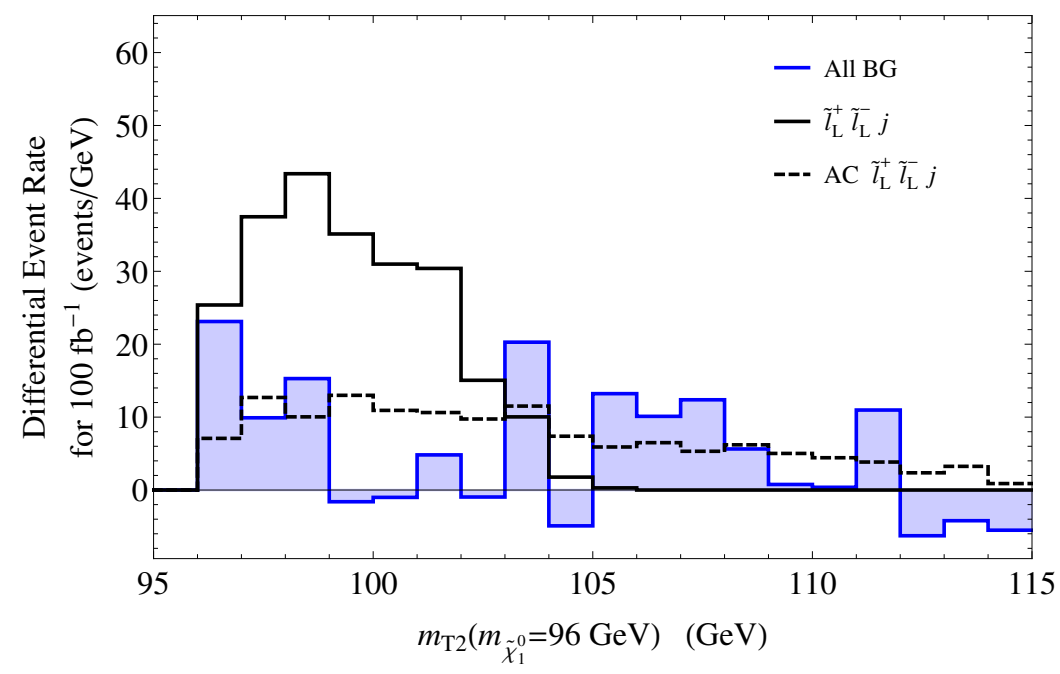

Figure 6. Different flavor subtracted $m_{\mathrm{T} 2}\left(p_{\mathrm{T}}^{l_{1}}, p_{\mathrm{T}}^{l_{2}} ; m_{\tilde{\chi}_{1}^{0}}\right)$ distribution after cuts $1-7$ for backgrounds and signal using $m_{\tilde{\chi}_{1}^{0}}=96 \mathrm{GeV}$ for both. Two different signal scenarios are shown: degenerate lefthanded selectrons and smuons with mass $m_{\tilde{l}_{L}}=104 \mathrm{GeV}$ each decaying to a lepton plus either a $96 \mathrm{GeV}$ neutralino LSP (solid black) or an auto-concealed modulino KK-tower (dashed black). Backgrounds are subtracted as described in section 3.3. The results are shown in this figure, not including the $m_{\mathrm{T} 2}$ cut described in step 8 .

statistical error in this expression as compared to its non-background subtracted counterpart eq. (3.5), the lower systematic error more than compensates for this and reduces the total uncertainty. For example, if we estimate $\sigma_{B \text { sys }}=0.2 B_{\mathrm{SF}}$ in (3.5), then for the typical backgrounds present in our study the background subtracted expression for $\sigma_{B}$ in eq. (3.6) reduces the total error by more than $60 \%$.

To summarize, we define our signal significance as $s=S / \sigma_{B}$ with $B=B_{\mathrm{SF}}-n B_{\mathrm{DF}}$ and $\sigma_{B}$ defined in eq. (3.6). An example of the signal as compared to the remaining background is shown in figure 6 .

\section{LHC14 reach with $100 \mathrm{fb}^{-1}$}

To evaluate the potential $95 \%$ confidence level (CL) exclusion reach of this analysis we demand a signal significance of 1.96. As figure 7 shows, LHC14 with $100 \mathrm{fb}^{-1}$ of data will be sensitive to sleptons with $3 \mathrm{GeV}<\Delta m<24 \mathrm{GeV}$ up to nearly $m_{\tilde{l}_{L}} \simeq 150 \mathrm{GeV}$. As can be seen from the figure, this analysis will allow the LHC to explore beyond $m_{\tilde{l}_{L}}>100 \mathrm{GeV}$ in the compressed region for the first time, though it will not completely close the gap between the compressed region and current ATLAS limits $(\Delta m \gtrsim 60 \mathrm{GeV})$. On the other hand, the production cross sections for right-handed sleptons are such that this analysis will only reach $m_{\tilde{l}_{R}}=100 \mathrm{GeV}$ with $100 \mathrm{fb}^{-1}$, and therefore will not improve on LEP2 limits; at least until the LHC has more data.

The analysis is constrained to work in the region $\Delta m \lesssim 24 \mathrm{GeV}$ because it relies on $m_{\mathrm{T} 2}$ to concentrate the signal events into a narrow mass range. The closer $m_{\tilde{l}}$ is to $m_{\tilde{\chi}_{1}^{0}}$ the more concentrated the signal events become and the better chance they have of beating 


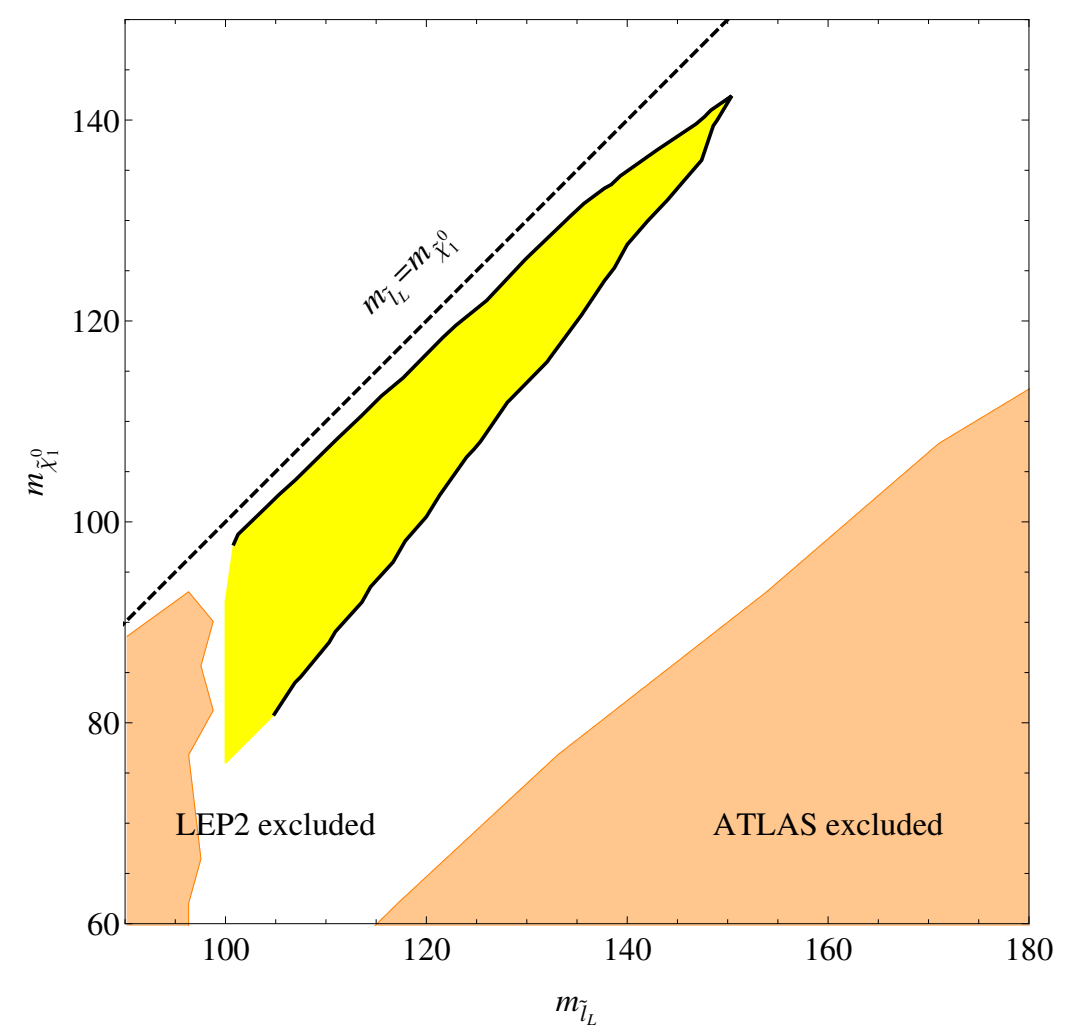

Figure 7. Potential 95\% CL exclusion reach at LHC14 with $100 \mathrm{fb}^{-1}$ for degenerate left-handed smuons and selectrons. Yellow area indicates the region excluded by this analysis, while orange areas indicate regions already excluded by LEP2 [31, 32] and ATLAS [2].

the backgrounds. However this only works down to about $\Delta m \simeq 3 \mathrm{GeV}$ because below this fewer leptons have enough energy to be tagged by the detector-even considering the fact that their parent sleptons are boosted from jet recoil.

In the case of auto-concealed SUSY detection becomes more challenging. Figure 8 shows the expected LHC14 $100 \mathrm{fb}^{-1}$ reach for excluding the production cross section of AC sleptons decaying to the KK-tower of a modulino LSP which lives in either $d=6$ or 3 extra dimensions. It will be able to exclude left-handed AC sleptons in $d=6$ (3) up to 110 (100) GeV. Again it will not be sensitive to right-handed AC sleptons except where LEP2 should have already been able to discover them.

Clearly, AC sleptons present a bigger detection challenge than a normal compressed spectrum. The reason for this is twofold. First, the largest branching ratios are to particles with masses $\sim 80 \%$ and $\sim 70 \%$ of the slepton mass for $d=6$ and 3 respectively (see figure 1). For $100 \mathrm{GeV}$ left-handed sleptons this puts $\Delta m$ just inside the reach of the analysis in the first case, and just outside in the second. As $m_{\tilde{l}}$ increases the effective $\Delta m$ only gets larger taking the model outside of the detection range. The second challenge is that the differential branching ratio distributions are quite broad. Therefore $m_{\mathrm{T} 2}$ is not able concentrate signal events into a well defined window starting at the LSP mass.

Despite these challenges, it is encouraging to see that for the auto-concealed scenarios the sensitivity of the analysis improves as the number $d$ of extra dimensions increases, in 

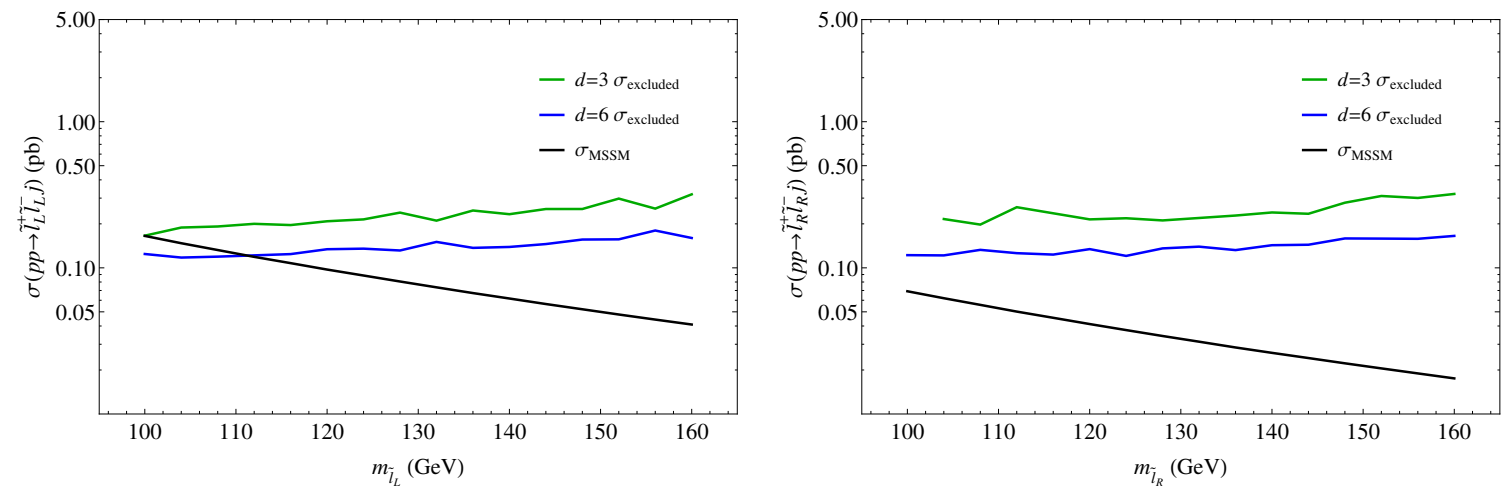

Figure 8. Potential 95\% CL exclusion reach at LHC14 with $100 \mathrm{fb}^{-1}$ for the production cross section of auto-concealed sleptons decaying to a modulino KK-tower in $d=3$ (green) or $d=6$ (blue) extra dimensions for left-handed (left plot) or right-handed (right plot) sleptons. The black line shows the tree level production cross section for $p p \rightarrow \tilde{l}^{+} \tilde{l}^{-} j$ indicating that, with this amount of data, the LHC should be sensitive to left-handed sleptons for $d=6$ (3) up to 110 (100) GeV. The production cross section of right-handed sleptons is smaller, requiring more data for sensitivity.

contrast to what was observed in ref. [17], where higher $d$ cases were more difficult. Higher integrated luminosities and/or reduced systematic uncertainties would further increase sensitivity.

\section{Conclusions}

We have presented an analysis built to detect sleptons with small $m_{\tilde{l}}-m_{\tilde{\chi}_{1}^{0}}$. We use the $p p \rightarrow \tilde{l}^{+} \tilde{l}^{-} j \rightarrow l^{+} l^{-} \tilde{\chi}_{1}^{0} \tilde{\chi}_{1}^{0} j$ channel, where the neutral, collider stable $\tilde{\chi}_{1}^{0} \mathrm{~s}$ are registered as $E_{\mathrm{T}}^{\text {miss }}$ and the jet (which is required to have $p_{\mathrm{T}}>100 \mathrm{GeV}$ ) comes from initial state radiation. The jet requirement distinguishes this search from 'classic' slepton analyses but is similar to monojet searches, and recent Higgsino search proposals [10, 11]. The jet requirement selects events with initial state radiation, and thus boosted sleptons. The resulting events tend to have large $E_{\mathrm{T}}^{\text {miss }}$ and daughter leptons with $p_{\mathrm{T}}$ above the detection threshold.

After a series of cuts to remove BG events a significant number of unwanted backgrounds remain, most notably from the leptonic decays of $t \bar{t}$ and $W^{+} W^{-} j$. Two final steps help to distinguish the signal from these processes. First, we take advantage of the 'stransverse' mass $m_{\mathrm{T} 2}$ to concentrate signal events into a window between the trial $m_{\tilde{l}}$ and $m_{\tilde{\chi}_{1}^{0}}$. Finally, we use the fact that signal leptons come in SFOS pairs while the major remaining backgrounds produce different flavor pairs as often as same flavor pairs. We use the different flavor pairs to 'subtract away' the majority of the remaining background. Though this introduces additional statistical error, this data-driven technique significantly reduces the systematic uncertainty and makes the signal detectable.

The analysis presented in this paper should allow LHC14 with $100 \mathrm{fb}^{-1}$ to search for degenerate left-handed selectrons and smuons in the compressed region $3 \mathrm{GeV}<m_{\tilde{l}_{L}}-m_{\tilde{\chi}_{1}^{0}}<$ $24 \mathrm{GeV}$ for $m_{\tilde{l}_{L}} \lesssim 150 \mathrm{GeV}$. This area is beyond LEP2 limits and currently unexplored by 
the LHC. In addition, it should be sensitive to the challenging case of auto-concealed lefthanded sleptons decaying to the KK-tower of a modulino LSP which lives in $d=6$ extra dimensions up to $m_{\tilde{l}_{L}} \lesssim 110 \mathrm{GeV}$. In both the compressed spectrum and auto-concealed scenarios this analysis will need more data to improve on LEP2 limits for right-handed sleptons.

\section{Acknowledgments}

We thank John March-Russell for the many useful discussions about theoretical considerations. We thank Mireia Crispin-Ortuzar, William Kalderon, William Fawcett, Claire Gwenlan, Koichi Nagai, and Jonathan Burr for their very helpful comments on this paper. We thank Juan Rojo, Ulrich Haisch, and Emanuele Re for useful discussions regarding simulations and backgrounds. We thank Jonathan Patterson for helping us with the Oxford Theory Computing Cluster. AJB gratefully acknowledges the support of U.K. Science and Technology Facilities Council, the IPPP (Durham), and Merton College, Oxford. JS gratefully acknowledges support from the United States Air Force Institute of Technology. The views expressed in this letter are those of the authors and do not reflect the official policy or position of the United States Air Force, Department of Defense, or the U.S. Government.

Open Access. This article is distributed under the terms of the Creative Commons Attribution License (CC-BY 4.0), which permits any use, distribution and reproduction in any medium, provided the original author(s) and source are credited.

\section{References}

[1] ATLAS and CMS collaborations, Supersymmetry searches at the LHC, arXiv:1211.3887 [INSPIRE].

[2] ATLAS collaboration, Search for direct production of charginos, neutralinos and sleptons in final states with two leptons and missing transverse momentum in pp collisions at $\sqrt{s}=8 \mathrm{TeV}$ with the ATLAS detector, JHEP 05 (2014) 071 [arXiv:1403.5294] [INSPIRE].

[3] CMS collaboration, Searches for electroweak production of charginos, neutralinos and sleptons decaying to leptons and $W, Z$ and Higgs bosons in pp collisions at 8 TeV, Eur. Phys. J. C 74 (2014) 3036 [arXiv:1405.7570] [INSPIRE].

[4] J.F. Gunion and S. Mrenna, A Study of SUSY signatures at the Tevatron in models with near mass degeneracy of the lightest chargino and neutralino, Phys. Rev. D 62 (2000) 015002 [hep-ph/9906270] [INSPIRE].

[5] H.K. Dreiner, M. Krämer and J. Tattersall, How low can SUSY go? Matching, monojets and compressed spectra, Europhys. Lett. 99 (2012) 61001 [arXiv:1207.1613] [INSPIRE].

[6] S. Mukhopadhyay, M.M. Nojiri and T.T. Yanagida, Compressed SUSY search at the $13 \mathrm{TeV}$ LHC using kinematic correlations and structure of ISR jets, JHEP 10 (2014) 012 [arXiv: 1403.6028] [INSPIRE].

[7] C. Han, A. Kobakhidze, N. Liu, A. Saavedra, L. Wu and J.M. Yang, Probing Light Higgsinos in Natural SUSY from Monojet Signals at the LHC, JHEP 02 (2014) 049 [arXiv: 1310.4274] [INSPIRE]. 
[8] ATLAS collaboration, Search for pair-produced third-generation squarks decaying via charm quarks or in compressed supersymmetric scenarios in pp collisions at $\sqrt{s}=8 \mathrm{TeV}$ with the ATLAS detector, Phys. Rev. D 90 (2014) 052008 [arXiv:1407.0608] [InSPIRE].

[9] CMS collaboration, Search for top squarks decaying to a charm quark and a neutralino in events with a jet and missing transverse momentum, CMS-PAS-SUS-13-009 (2014) [INSPIRE].

[10] H. Baer, A. Mustafayev and X. Tata, Monojet plus soft dilepton signal from light higgsino pair production at LHC14, Phys. Rev. D 90 (2014) 115007 [arXiv:1409.7058] [INSPIRE].

[11] Z. Han, G.D. Kribs, A. Martin and A. Menon, Hunting quasidegenerate Higgsinos, Phys. Rev. D 89 (2014) 075007 [arXiv: 1401.1235] [INSPIRE].

[12] P. Schwaller and J. Zurita, Compressed electroweakino spectra at the LHC, JHEP 03 (2014) 060 [arXiv: 1312.7350] [INSPIRE].

[13] C.G. Lester and D.J. Summers, Measuring masses of semi-invisibly decaying particles pair produced at hadron colliders, Phys. Lett. B 463 (1999) 99 [hep-ph/9906349] [INSPIRE].

[14] A. Barr, C.G. Lester and P. Stephens, A variable for measuring masses at hadron colliders when missing energy is expected; $m_{T 2}$ : the truth behind the glamour, J. Phys. G 29 (2003) 2343 [hep-ph/0304226] [INSPIRE].

[15] H.-C. Cheng and Z. Han, Minimal Kinematic Constraints and $m_{T 2}$, JHEP 12 (2008) 063 [arXiv:0810.5178] [INSPIRE].

[16] CMS collaboration, Search for physics beyond the standard model in events with two opposite-sign same-flavor leptons, jets and missing transverse energy in pp collisions at $\sqrt{s}=8 \mathrm{Te}$, CMS-PAS-SUS-12-019 (2014) [INSPIRE].

[17] S. Dimopoulos, K. Howe, J. March-Russell and J. Scoville, Auto-Concealment of Supersymmetry in Extra Dimensions, arXiv:1412.0805 [INSPIRE].

[18] B. Dutta et al., Probing Compressed Sleptons at the LHC using Vector Boson Fusion Processes, Phys. Rev. D 91 (2015) 055025 [arXiv: 1411.6043] [InSPIRE].

[19] Z. Han and Y. Liu, $M_{T 2}$ to the Rescue - Searching for Sleptons in Compressed Spectra at the LHC, arXiv: 1412.0618 [INSPIRE].

[20] J. Alwall et al., The automated computation of tree-level and next-to-leading order differential cross sections and their matching to parton shower simulations, JHEP 07 (2014) 079 [arXiv: 1405.0301] [INSPIRE].

[21] T. Sjöstrand, S. Mrenna and P.Z. Skands, PYTHIA 6.4 Physics and Manual, JHEP 05 (2006) 026 [hep-ph/0603175] [INSPIRE].

[22] O. Shekhovtsova, I.M. Nugent, T. Przedzinski, P. Roig and Z. Was, MC generator TAUOLA: implementation of Resonance Chiral Theory for two and three meson modes. Comparison with experiment, AIP Conf. Proc. 1492 (2012) 62 [arXiv:1208.5420] [INSPIRE].

[23] M. Drees, H.K. Dreiner, D. Schmeier, J. Tattersall and J.S. Kim, CheckMATE: Confronting your Favourite New Physics Model with LHC Data, Comput. Phys. Commun. 187 (2015) 227 [arXiv: 1312.2591] [INSPIRE].

[24] M. Cacciari and G.P. Salam, Dispelling the $N^{3}$ myth for the $k_{t}$ jet-finder, Phys. Lett. B 641 (2006) 57 [hep-ph/0512210] [INSPIRE].

[25] A.L. Read, Presentation of search results: The $C L_{s}$ technique, J. Phys. G 28 (2002) 2693 [INSPIRE]. 
[26] DELPHES 3 collaboration, J. de Favereau et al., DELPHES 3, A modular framework for fast simulation of a generic collider experiment, JHEP 02 (2014) 057 [arXiv:1307.6346] [INSPIRE].

[27] M. Cacciari, G.P. Salam and G. Soyez, FastJet User Manual, Eur. Phys. J. C 72 (2012) 1896 [arXiv: 1111.6097] [INSPIRE].

[28] M. Cacciari, G.P. Salam and G. Soyez, The Anti-k $k_{t}$ jet clustering algorithm, JHEP 04 (2008) 063 [arXiv: 0802.1189] [INSPIRE].

[29] ATLAS collaboration, Search for direct-slepton and direct-chargino production in final states with two opposite-sign leptons, missing transverse momentum and no jets in 20/fb of pp collisions at $\sqrt{s}=8 \mathrm{TeV}$ with the ATLAS detector, ATLAS-CONF-2013-049 (2013) [INSPIRE].

[30] G. Cullen, N. Greiner and G. Heinrich, Susy-QCD corrections to neutralino pair production in association with a jet, Eur. Phys. J. C 73 (2013) 2388 [arXiv:1212.5154] [INSPIRE].

[31] LEP2 SUSY Working Group, Combined LEP Chargino Results, up to $208 \mathrm{GeV}$ for large m0, LEPSUSYWG/01-03.1 (2004) and online at http://lepsusy.web.cern.ch/lepsusy/www/inos_moriond01/charginos_pub.html.

[32] LEP2 SUSY Working Group, M. Berggren et al., Combined LEP Selectron/Smuon/Stau Results, 183-208 GeV, LEPSUSYWG/04-01.1 (2004) and online at http://lepsusy.web.cern.ch/lepsusy/www/sleptons_summer04/slep_final.html. 